\title{
Microscopic Estimation of Road Impedance by Decomposing Traffic Delay into Individual Road Segments: An Analytical Approach
}

\author{
Jiang Mi, ${ }^{1,2}$ Yuelong Bai ${ }^{D},{ }^{1}$ Mingbo Luo ${ }^{D},{ }^{1}$ Hui Wang $\mathbb{D}^{2},{ }^{2}$ and Minrui Chen ${ }^{3}$ \\ ${ }^{1}$ School of Transportation and Logistics, East China Jiaotong University, Nanchang 330013, China \\ ${ }^{2}$ Jiangxi Transportation Institute, Nanchang 330200, China \\ ${ }^{3}$ Jiangxi Provincial Highway Network Management Center, Nanchang 330036, China \\ Correspondence should be addressed to Yuelong Bai; yuelong@ecjtu.edu.cn
}

Received 18 January 2019; Accepted 12 March 2019; Published 2 May 2019

Academic Editor: Paolo Lonetti

Copyright ( 2019 Jiang Mi et al. This is an open access article distributed under the Creative Commons Attribution License, which permits unrestricted use, distribution, and reproduction in any medium, provided the original work is properly cited.

\begin{abstract}
Urban road dynamic impedance calculation is the important basis of dynamic traffic assignment and real-time traffic management and control scheme. The current impedance calculation is mostly based on BPR function, and the BPR function is derived from macroscopic statistical laws, in which the microscopic characteristics of traffic flow are insufficiently described. In order to more accurately express the change laws of traffic impedance at the microscopic level, a stochastic dynamic traffic assignment algorithm based on road impedance function is designed to analyze the time impedance of congested roads under random dynamic traffic assignment under different conditions of road network saturation. Compared with the current model, the comprehensiveness and portability are greatly improved. The results show that the impedance calculation error of this method is less than $10 \%$ when the load degree of the road is lower than 0.85 , which proves that the method has good precision under unsaturated flow conditions.
\end{abstract}

\section{Introduction}

The current urban traffic congestion problem has become a social issue that both urban ordinary travelers and urban managers are concerned about. The development of the city is affected by traffic congestion, which also affects the travel of urban residents. Each traveler spends more and more time on the delays caused by traffic congestion. In traffic assignment, the traffic impedance function plays an important role[1], and the traffic impedance is a measure value that expresses the magnitude of the impedance (i.e., the degree of obstruction or the degree of traffic difficulty) encountered by a vehicle in the course of running. And it is a measurement that expresses the magnitude of the accessibility and smoothness of the vehicle in running. Traffic impedance is the basic element of path impedance, which directly affects travelers' path choice behavior [2] and is of great significance to traffic allocation of urban road network [3].

The impedance function is mainly composed of two parts: road impedance and node impedance [4], in which the road impedance is an important parameter for traffic assignment and road network planning, and it is also the focus of research on OD flow fitting problem [5]. The generalized road impedance (also known as delay, cost) is the resistance of people, cars, and roads to traffic travel. The narrowly defined road impedance generally refers to the travel time spent by the vehicle on the road. The dynamic traffic bottleneck in the urban road network is the bottleneck caused to the nodes or roads in the road network under the condition that the road network conditions are unchanged and the traffic flow changes in real time (dynamic). This paper uses travel time as the main reference standard for traffic impedance.

At present, the widely used road impedance measurement method is the BPR function proposed by BPR (U.S. Bureau of Public Roads) [6]. Spiess [7] corrected the BPR function for the problem of too low precision caused by too high parameter value in the BPR function. Based on the study of Spiess [7], Zudhy et al. [8] also improved the BPR function model to solve the problem of small change in running time when the saturation is low. Davidson [8] proposed 
a road impedance calculation method in congestion and noncongestion state by using queuing theory. Tan et al. [9] and Lin et al. [10] found that, by classifying the roads with different traffic congestion conditions to estimate road impedance, they achieved better results than the traditional BPR function. Some studies [4] believe that the road environment will also affect the traffic impedance function. Yan et al. [4] proposed a comprehensive model of urban road impedance based on linear regression after analyzing the data of urban road sections and intersections. Dong et al. [5] considered the influence of road length and speed, road elevation, traffic lights, and waiting time at intersections on the road impedance function. Abolghasem et al. [11] proposed an impedance model based on analytic hierarchy process (AHP) by combining the influence of weather, sightseeing information, road type, and other variables on the change of road impedance. Other literatures [12] believe that the characteristics of the traffic flow also have a great influence on the traffic impedance function. Luo et al. [12] take signalized intersections as the research object, and, based on traditional traffic wave theory, they use basic driving time and waiting delay time to build the traffic impedance function. He et al. [13] believed that intersection density, bus station density, nonmotor vehicle density, road saturation, and other factors would also have an impact on road traffic impedance. Cai et al. [14] proposed a traffic impedance function based on the grey average generation function and determined the relationship between traffic flow and travel time by using the relationship among traffic flow, velocity, and density. Since the change of urban road obstruction will affect the planning of people's travel route [15], the estimation of road impedance should also be dynamic. Jin et al. [16] analyzed the dynamic change laws of road congestion at the road bottleneck based on kinematic wave theory. Sun et al. [17] estimated the road impedance in real time by analyzing the dynamic changes of the intersection period. Qu et al. [18], in order to improve the coordination of traffic signal settings and distribution, considered the effect of the dynamic impedance path for people travel plan. The existing dynamic impedance model has a single structure and cannot adapt to the road impedance estimation needs under different traffic flow distributions. In addition, the impedance estimation error under congested state is large, and the applicable range is limited.

Therefore, the aim of this paper is mainly to construct the road dynamic impedance function model. After constructing the virtual road network in the target area, the key point for identifying it is to load different traffic flows into the road network and construct the dynamic road impedance function model to perform dynamic traffic assignment for the loaded traffic flow, so as to identify the road network bottleneck states under different traffic demands. A random dynamic traffic assignment algorithm is designed based on the road impedance function. By analyzing the road network saturation achieved by different traffic flows under random dynamic traffic assignment, the road network dynamic bottleneck state is determined finally and the forming process thereof analyzed. The road impedance function based on traffic flow is constructed by travel time, and good results are obtained by simulation software. This paper solves the traditional construction problems of the road impedance function model, and its comprehensiveness, real-time performance, and portability are greatly improved. Therefore, the dynamic road impedance function model proposed in this paper can be applied to the determination of the travel time impedance of the vehicle in crowded road conditions and provides a path impedance basis for dynamic traffic assignment.

The rest of this paper is organized as follows. Section 2 provides Dynamic Impedance Modeling Method. Section 3 introduces numerical experiment. In Section 4 the experimental results are discussed. The conclusion is given in Section 5.

\section{Methodology}

In order to conduct dynamic traffic assignment, it is especially critical to accurately determine the road impedance function of the traffic flow in the path of the road network. The traditional road impedance function is mainly to simply superimpose the travel time of the traffic flow on the road segment between the upstream and the downstream intersections and the delay at the downstream intersection but not to analyze the specific form of the segment traffic flow (intermittent flow) during running and the specific process of queuing in front of the intersection. In this section, our proposed model will consider the specific queuing process of the traffic flow at the intersection and perform queuing clearance analysis to obtain a more accurate road impedance function considering the queue length of the vehicle. Because the queue length of the vehicle at the intersection changes in real time, the obtained road impedance function is also dynamic.

2.1. Determination of Road Impedance Function. The road impedance function of traffic, also known as impedance function, is an indispensable concept in road network traffic flow assignment and includes many composition factors, such as road travel time (narrow sense), road traffic saturation, intersection delay, intersection load, passenger comfort, and travel cost. In addition, this impedance can be regarded as road expense (generalized). In the road expense, time is what we mainly consider. Therefore, the time is selected as the measurement standard in this chapter, and the road impedance function constructed in this chapter is the function of the road travel time. There are several reasons for using time as a measurement standard:

(1) Experience shows that time is the most important factor affecting traffic flow on road segments.

(2) Other impedance composition factors are closely related to the time measurement index and they have the same direction rise and fall relationship.

(3) Time is undoubtedly the easiest to measure relative to other impedance composition factors, which is convenient for practical applications. In practical applications, other factors are often replaced with time measurement index in a broad sense. 


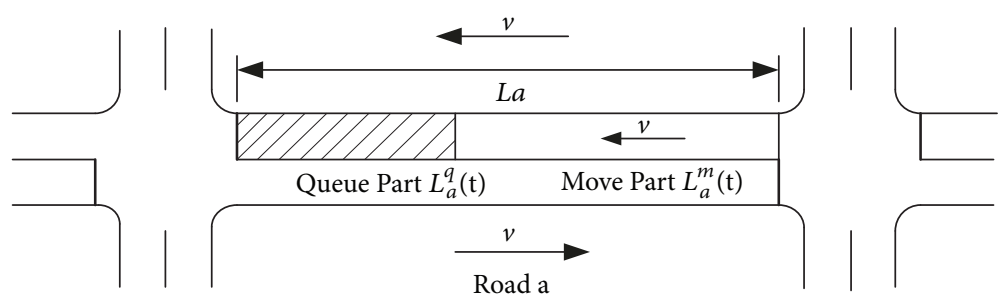

FIGURE 1: Section impedance-travel time composition analysis.

2.2. Road Impedance Function-Travel Time Composition Study. The travel time of the traffic flow on the road segment is the travel time spent on the road segment for the continuous traffic flow, but this situation is obviously not realistic. In the urban road network, due to the existence of the intersections and the setting of the signal lights, the traffic flow is intermittent, and the traffic flow on the crowded road segment presents two traffic flow states, one of which is driving state and the second of which is queue state. According to the traffic flow theory, both the "travel distance" and the "average travel speed" determine the travel time of the intermittent traffic flow on the road segment. Therefore, this section divides the segment between the upstream and the downstream intersections into two parts: one is the driving part and the second is the queuing part.

As shown in Figure 1, the vehicle travels on the segment $a$ between the upstream and the downstream intersections. The traffic flow leaves the upstream intersection at the speed of $v_{a}^{m}(t)$, and, when entering the downstream intersection, the vehicle is in queue due to the influence of the signal light. Assuming the queue length is $L_{a}^{q}(t)$, the distance traveled on the road segment $a$ is $L_{a}^{m}(t)$, and the total length of the road segment is $L_{a}$; then

$$
L_{a}=L_{a}^{m}(t)+L_{a}^{q}(t)
$$

In which $v_{a}^{m}(t), L_{a}^{m}(t)$, and $L_{a}^{q}(t)$ are all time functions.

Similarly, it is assumed that the time taken by the vehicle to travel on segment $a$ is $T_{a}^{m}(t)$, the delay caused by the queue (including stop and go) is $T_{a}^{q}(t)$, and the total travel time on section $a$ is $T_{a}(t)$, so

$$
T_{a}(t)=T_{a}^{m}(t)+T_{a}^{q}(t)
$$

In which

$$
T_{a}^{m}(t)=\frac{L_{a}^{m}(t)}{v_{a}^{m}(t)}=\frac{L_{a}-L_{a}^{q}(t)}{v_{a}^{m}(t)}
$$

It can be seen from Equations (2) and (3) that, in order to obtain the road impedance $T_{a}(t)$, it is necessary to separately determine the travel time $T_{a}^{m}(t)$ of the travel segment and the delay time $T_{a}^{q}(t)$ of the queue part. The travel time $T_{a}^{m}(t)$ can be considered from the queue length $L_{a}^{q}(t)$. Therefore, to determine $T_{a}^{m}(t)$ is converted into determining the real-time queue length $L_{a}^{q}(t)$, and it can be analyzed and verified by gather-disperse wave.
2.3. Analysis of Gather-Disperse Wave at Queue. The gatherdisperse wave analysis is derived from the traffic wave theory, which considers the flow as a continuous fluid to simulate this continuous fluid and establish a continuous equation. According to the known knowledge of the traffic wave theory, the equation for calculating the queuing wave velocity $\omega$ is

$$
\omega=\frac{x}{t}=-\frac{l_{1}}{t}+v_{1}=\frac{l_{2} v_{1}-l_{1} v_{2}}{l_{2}-l_{1}}=\frac{v_{1} / k_{2}-v_{2} / k_{1}}{1 / k_{2}-1 / k_{1}}
$$

In order to accurately characterize the queuing and disperse process of the vehicle in front of the intersection, the famous Greenshields model is used here:

$$
v_{i}=v_{f}\left(1-\frac{k_{i}}{k_{j}}\right)
$$

In the equation $v_{i}$ is the instantaneous speed $(\mathrm{km} / \mathrm{h}), i \in$ $t$ at any moment $i$ within time $t ; k_{i}$ is the traffic density $(v e h / \mathrm{km}), i \in t$ on the road segment at any moment $i$ within time $t ; v_{f}$ is free flow speed $(\mathrm{km} / \mathrm{h})$ on the road segment; and $k_{j}$ is the congestion density $(v e h / \mathrm{km})$ on the road segment.

Another calculation method of traffic waves can be obtained from the queuing wave velocity calculation equation and the Greenshields model:

$$
\omega=v_{f}\left(1-\frac{k_{1}+k_{2}}{k_{j}}\right)
$$

When the traffic flow is in queue at the intersection and changes from a certain density traffic flow to a traffic flow (stop to queue) with a congestion density zero speed, the stop of the first car will cause the second car and the subsequent cars to stop. This kind of wave, which is opposite to the driving direction of traffic flow, is called a stop wave. After the vehicle is stopped, its density is the max, reaching the congestion density $k_{j}$ (i.e., $k_{2}=k_{j}$ ). According to Equation (6), the calculation equation of the wave velocity $\omega_{\text {stop }}$ of the stop wave is

$$
\omega_{s t o p}=-v_{f} \frac{k_{1}}{k_{j}}
$$

The simultaneous Equations (5) and (7) eliminate $v_{f}$ to obtain

$$
\omega_{\text {stop }}=-\frac{q}{k_{j}-k_{i}}
$$


2.4. Determination of Dynamic Impedance Model Equation. It is known from Section 2.2 that to determine the travel time $T_{a}^{m}(t)$ (part of the road impedance) of the travel part on the road segment is actually converted into determining the crowded queue length $L_{a}^{q}(t)$ on the road segment. According to the gather-disperse analysis of the traffic flow in Section 2.3, it can be seen that the traffic flow changes from the high-speed low-density state to the zero-speed congestion density state before queuing, and, at the same time, a stop wave opposite to the driving direction is generated. It is assumed that the queuing occurs on the road segment $a$, the stop wave velocity is $\omega_{\text {stop, } a}$, and the driving direction is the positive direction, so the stop to queue length is

$$
L_{a}^{q}(t)=d_{t} \omega_{\text {stop,a }}=-d_{t} v_{f} \frac{k_{1}}{k_{j}}
$$

In the equation, $d_{t}$ is the vehicle queue delay time caused by the signal light at the downstream intersection. In Equation (1), the parking wave velocity is negative, and the queue length is positive, so the queue length must be determined by taking the absolute value of the wave velocity; (2) in order to ensure the uniformity of the variables, the real-time density $k_{1}$ of the traffic flow in the motion part is replaced with $k_{a}^{m}(t)$; at this time $k_{2}=k_{j}$. So the queue length, Equation (9) is transformed as follows:

$$
L_{a}^{q}(t)=d_{t}\left|\omega_{\text {stop, } a}\right|=d_{t} v_{f} \frac{k_{a}^{m}(t)}{k_{j}}
$$

To calculate the queue length $L_{a}^{q}(t)$ on the road segment $a$, it can be seen from Equation (10) that it is a function with " $k_{a}^{m}(t)$ " as a variable.

For the road segment length $L_{a}^{m}(t)$ of the motion part, the real-time speed of the vehicle can be regarded as the ratio between the inflow rate $I_{a}(t)$ and $L_{a}^{m}(t)$ and the real-time traffic density $k_{a}^{m}(t)$ at the upstream intersection to the road segment $a$; the greenshields model is used, namely,

$$
v_{a}^{m}(t)=\frac{I_{a}(t)}{k_{a}^{m}(t)}=v_{f}\left(1-\frac{k_{a}^{m}(t)}{k_{j}}\right)
$$

For $I_{a}(t) / k_{a}^{m}(t)=v_{f}\left(1-k_{a}^{m}(t) / k_{j}\right)$, it can be obtained that this equation is a univariate quadratic equation with " $k_{a}^{m}(t)$ " as a variable. Combined with Equation (10), the variable $k_{a}^{m}(t)$ is eliminated to obtain the crowded queue length $L_{a}^{q}(t)$ of the vehicle on the road segment $a$ :

$$
L_{a}^{q}(t)=\frac{d_{t} v_{f}}{2}\left|1 \pm \sqrt{1-\frac{4 l I_{a}(t)}{v_{f}}}\right|
$$

In the equation, $l$ is the average standard length $(\mathrm{km})$ of single vehicle, $l=1 / k_{j}$ (replace the congestion density $k_{j}$ ).

At this point, the crowded queue length $L_{a}^{q}(t)$ on the road segment $a$ is finally determined. Ignore the stop to queue interval of the vehicle; for the queuing vehicle number $M=$ $L_{a}^{q}(t) / l$, the number of real-time queuing vehicles in a single lane is

$$
M_{a}(t)=\frac{d_{t} v_{f}}{2 l}\left|1 \pm \sqrt{1-\frac{4 l I_{a}(t)}{v_{f}}}\right|
$$

This paper adopts $v_{a}^{m}(t) \approx v_{f}$ and combines Equations (12) and (3) to obtain the road impedance $T_{a}^{m}(t)$ of the driving part:

$$
\begin{aligned}
T_{a}^{m}(t) & =\frac{L_{a}^{m}(t)}{v_{a}^{m}(t)}=\frac{L_{a}-L_{a}^{q}(t)}{v_{a}^{m}(t)} \\
& =\frac{L_{a}-d_{t} v_{f}\left|1 \pm \sqrt{1-4 l I_{a}(t) / v_{f}}\right|}{2 v_{f}}
\end{aligned}
$$

According to Equations (2) in Section 2.2, the impedance function proposed in this paper consists of two parts: the driving part road impedance $T_{a}^{m}(t)$ and the crowded queue road impedance $T_{a}^{q}(t)$. For the fixed road length $L_{a}$, it can be seen from Equations (1) and (2) that the real-time driving part road length $L_{a}^{m}(t)$ and the crowded queue length $L_{a}^{q}(t)$ are a whole that is interrelated and mutually influential, so the impedance of the two parts is not independent of each other. Both can be seen as a linear function relationship in general.

For solving the crowded queue road impedance $L_{a}^{q}(t)$, $L_{a}^{q}(t)$ is the ratio between the number $M_{a}(t)$ of the vehicles queuing in front of the downstream intersection at the moment $t$ and the real-time flow outgoing rate $C_{\text {down,a }}(t)$ at the downstream intersection at the moment $t$; that is,

$$
\begin{aligned}
T_{a}^{q}(t) & =\frac{M_{a}(t)}{C_{\text {down, } a}(t)} \\
& =\frac{d_{t} v_{f}}{2 l C_{\text {down, } a}(t)}\left|1 \pm \sqrt{1-\frac{4 l I_{a}(t)}{v_{f}}}\right|
\end{aligned}
$$

The dynamic impedance model equation on the road segment $a$ can be obtained from Equations (2), (14), and (15):

$$
\begin{aligned}
T_{a}(t)= & \frac{L_{a}-d_{t} v_{f}\left|1 \pm \sqrt{1-4 l I_{a}(t) / v_{f}}\right|}{2 v_{f}} \\
& +\frac{d_{t} v_{f}}{2 l C_{\text {down, } a}(t)}\left|1 \pm \sqrt{1-\frac{4 l I_{a}(t)}{v_{f}}}\right|
\end{aligned}
$$

2.5. Defining Impedance for the Travel Path of Road Net. The path $k$ altogether comprises $x$ stretches; that is, $k_{m \rightarrow n}=$ $\left\{a_{1}, a_{2}, a_{3}, \ldots, a_{x-1}, a_{x}\right\}$. The dynamic impedance of the path $k$ can be obtained according to the dynamic impedance equation of stretch defined in the last section.

Assume the traveler starts from the origin (road net node $m$ ) at time $\tau$ for destination node $n$; then the time for him/her to reach the stretches along the starting path $k$ is

$$
\tau_{a_{1}}=\tau
$$

The time to reach the stretches $a_{2}$ is the starting time plus the travel impedance on the stretch $a_{1}$. According to Equation (16)

$$
\tau_{a_{2}}=\tau_{a_{1}}(\tau)+T_{a_{1}}\left(\tau_{a_{1}}(\tau)\right)
$$




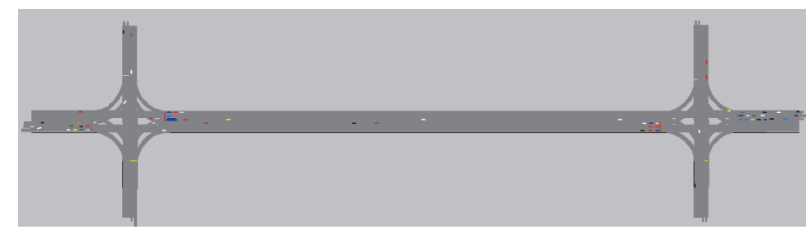

Figure 2: Simulation scene.

Similarly

$$
\tau_{a_{3}}=\tau_{a_{2}}(\tau)+T_{a_{2}}\left(\tau_{a_{2}}(\tau)\right)
$$

The time for the traveler to reach the stretch $x$ along the travel path $k$ can be derived according to the induction recursion method:

$$
\tau_{a_{x}}=\tau_{a_{x-1}}(\tau)+T_{a_{x-1}}\left(\tau_{a_{x-1}}(\tau)\right)
$$

It is observed from Equation (16) Equation (20) that the dynamic travel path (transient impedance) on path $k$ is the sum of dynamic impedance for all the stretches on the path. The research in the previous sections shows that the given travel impedance for the stretches is composed of queuing time, nonqueuing time, and the flow $q$ on the stretch. The real-time outflow rate of vehicle stream at the downstream junction $C_{\text {down,a }}(\tau)$ and the real-time inflow rate of vehicle stream at the upstream junction $I_{a}(\tau)$ serve as the variables. The real-time inflow rate of vehicle stream at the upstream junction $I_{a}(\tau)$ serves as the variable. Thus, the transient travel impedance $\mathscr{R}$ of the path is the function of $C_{\text {down, } a}(\tau), I_{a}(\tau)$ and $q_{a}(\tau)$; that is,

$$
\mathscr{R}(\tau)=f\left(C_{\text {down,a }}(\tau), I_{a}(\tau), q_{a}(\tau)\right)
$$

Discretize it to obtain

$$
\mathscr{R}_{k}^{m \longrightarrow n}(\tau)=\sum_{a=1}^{x} T_{a}\left(C_{\text {down,a }}(\tau), I_{a}(\tau), q_{a}(\tau)\right)
$$

Now the model of dynamic travel impedance is established.

\section{Case Study}

To verify the above-established path impedance function model, this section adopts the Vissim software to simulate the transportation flow of the stretch and obtain the average travel time of vehicle stream on the stretch, thereby verifying the established dynamic impedance function.

The simulation via the Vissim software mainly has the following advantages. (1) The software is a microcosmic simulation modeling tool based on the time interval and "psychological-physiological driving behavior model" used for transportation modeling simulation of urban transportation and public transportation running. (2) It is able to create model and analyze the transportation performance in various transportation conditions (lane setting, transportation composition, traffic signal, bus stop, etc.). (3) It adopts discrete and random microcosmic model with step of $0.1 s$. The longitudinal movement of vehicle is based on rule algorithm. (4) It provides graphic interface, adopts the 2D and $3 \mathrm{D}$ animation effect to visually display the vehicle motion to the user, and applies the dynamic transportation distribution to select the path.

3.1. Establish the Simulation Scenario. Before establishing the simulation scenario, the detected stretch also needs to be defined here to calculate the average travel time of stretch besides above parameters setting. This section defines the detected stretch: the detected stretch between the second travel time detector behind the stop line of downstream junction and the first travel time detector at upstream $500 \mathrm{~m}$ from the first detector along the stretch; it is composed of one origin and one destination. The length of this detected region can be regarded as the length $500 \mathrm{~m}$ of the stretch between two junctions in the real road net. The average travel time here refers to the time interval between when the vehicle passes the origin of the detector and when the vehicle leaves the destination of detector. Thus, here it comprises two parts: the time for driving and the time for stopping and waiting.

Setting of parameters for the travel time detector, flow loading, design of signal timing plan, parameter setting for output file "travel time," and simulation scenario are as shown in Figure 2.

This simulation scenario is established with the routine crossroad as the model and simultaneously considers the two upstream and downstream junctions. With the stretches between the two junctions as the carrier of research, the establishing considers byway transportation flow to mainly research and analyze the one-way transportation flow. Via the above research method, the travel time for the vehicle flow on this stretch is analyzed in a simulative way.

3.2. Setting of Simulation Parameters. The simulation here is to verify the feasibility and applicability of the dynamic impedance function model proposed above. The model is evaluated via a comparative analysis of the simulation result and the calculation result of function model. As the above dynamic impedance function is expressed as the impedance function for a certain stretch in the road net, this simulation is on a certain stretch. Before establishing the simulation scenario, the simulation parameters of stretch should be set.

When we use Vissim to do the road segment simulation environment, set the length of the link between the upstream and the downstream intersections to $0.5 \mathrm{~km}$ (the length of the detection area in the simulation scene), and set it to twoway six lanes. Create the vehicle input and type (converted 
TABLE 1: Simulation parameters setting.

\begin{tabular}{lcccc}
\hline Road Length & $\begin{array}{c}\text { Avg of Vehicle } \\
L_{a}(\mathrm{~km})\end{array}$ & $\begin{array}{c}\text { Length } \\
l(\mathrm{~km})\end{array}$ & $\begin{array}{c}\text { Velocity of } \\
\text { Free-flow } \\
v_{f}(\mathrm{~km} / \mathrm{h})\end{array}$ & $\begin{array}{c}\text { Outflow Rate of } \\
\text { Downstream } \\
C_{\text {down,a }}(\text { veh } / h)\end{array}$ \\
\hline Value & 0.5 & 0.005 & 50 & 6000 \\
\hline
\end{tabular}

into standard vehicles with length of $0.005 \mathrm{~km}$ ). Set the freeflow velocity of vehicles as $50 \mathrm{~km} / \mathrm{h}$ and the smallest safe time-distance as $(1 / 2000) h$, so the saturated traffic capacity of each lane $C_{\text {down, }}(t)$ is $2000 v e h / h$. As the signal cycle at the junctions in urban road net is advisable to be $50 s \sim 90 s$, and $100 s \sim 120 s$ in rush hours, and to not exceed $140 s$, we adopt the design value of $140 \mathrm{~s}$ for the largest signal cycle and $(1 / 90) h$ for corresponding red light to make the simulation universal (including the traffic flow in rush hours). Then the concrete simulation parameters setting is as shown in Table 1.

After the simulation parameters are set, it is needed to load flow to the simulation scenario to be used as the vehicle inflow rate $I_{a}(t)$ to the stretch $a$ from the upstream junction. As the simulated object is dynamic transportation flow, its traffic volume is changing with time. Assume that the traffic inflow rate on stretch $a$ at time $t$ is $I_{a}(t)$ and the traffic inflow rate on stretch $a$ at time $t+1$ is $I_{a}(t+1)$ ( 1 is the unit time length, here referring to the next transient time of time $t$ ); then $\Delta I=I_{a}(t+1)-I_{a}(t)$. In the real world, the traffic inflow rate is changing, and its increment $\Delta I$ can be greater than, smaller than, or equal to zero. However, the paper can macroscopically ignore the fine changes of traffic flow rate at transient time to verify the feasibility and applicability of the dynamic impedance function model proposed above, thereby discretizing the real-time dynamic traffic flow to just verify the application scope of this mode.

For the traffic flow loading in the simulation scenario, this section considers increasing the traffic flow isochronally and isometrically by loading traffic volume of $400 \mathrm{veh} / \mathrm{h}$ in the beginning and increasing $50 v e h / h$ every $10 \mathrm{~min}$. The loading of traffic flow is as shown in Table 2.

The flow is loaded to the stretch in the simulation scenario with the method of traffic flow loading as shown in Figure 2. Set the time range for output of simulation result as $60 \mathrm{~s}$ and simulation time range as 3600 s. Considering the output result of the first 600 s by the Vissim software is instable, here we select the simulation result data of $600 \sim 3600$ s for analysis and evaluation to eliminate the instability factors of the system. Therefore, we take 5 groups of traffic volume for one hour for a single simulation scenario and 10 groups of result data for each group of traffic volume.

\section{Results and Discussions}

The flow is loaded to the simulation scenario by beginning the transportation flow from $400 v e h / h$ and increasing $50 v e h / h$ every $10 \mathrm{~min}$. The travel time scope obtained from simulation on different transportation flows is predicted with $60 \mathrm{~s}$ as the output time period of simulation result. Then the result is compared with the calculation result by the dynamic impedance function model proposed in Section 2.4, to define

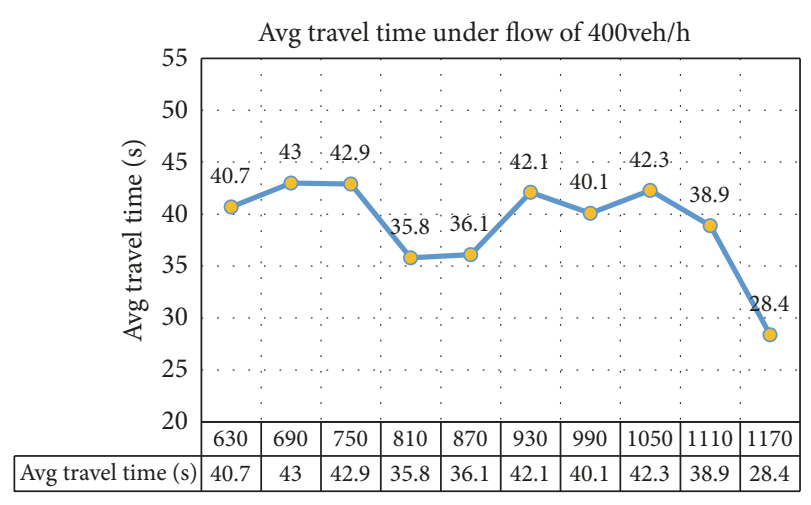

Time line 600 1200 (s)

Figure 3: Travel time line chart (Flow 400veh/h).

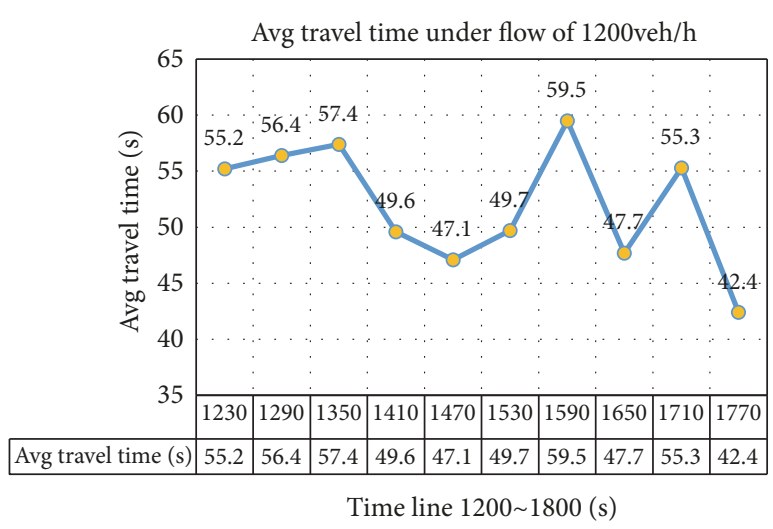

FIgURE 4: Travel time line chart (Flow 1200veh/h).

its application scope. Part of simulation data is as shown in Figures 3-6.

Although the transportation inflow rate is dynamic, here we can macroscopically ignore the fine changes of transportation flow rate at transient time to verify the feasibility and applicability of the dynamic impedance function model proposed above, thereby discretizing the real-time dynamic traffic flow to just verify the application scope of this mode. Thus, the simulation data of the first 600s is removed (lest the system instability should lead to data unreliability). In our simulation data, we set the simulated traffic volume at $600 \sim 1200$ s as $400 v e h / h, 1200 \sim 1800 s$ as $1200 v e h / h, 2400 \sim$ $3000 \mathrm{~s}$ as $1550 v e h / h$, and $3000 \sim 3600 s$ as $1850 v e h / h$. Figure 3 shows the experimental road average travel time changes in $600 \sim 1200$ s, Figure 4 shows the experimental road average travel time changes in 1200 1800s, Figure 5 shows the experimental road average travel time changes in 2400 3000s, and Figure 6 shows the experimental road average 


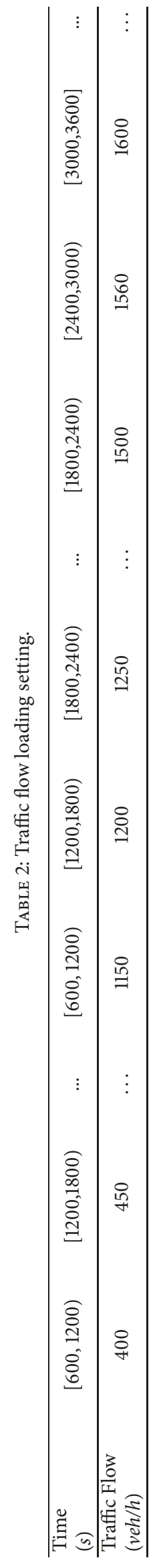




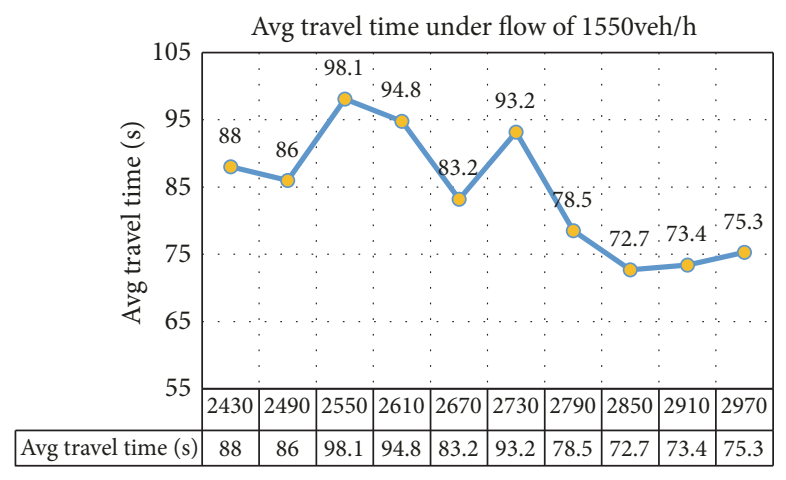

Time line 2400 3000 (s)

Figure 5: Travel time line chart (Flow 1550veh/h).

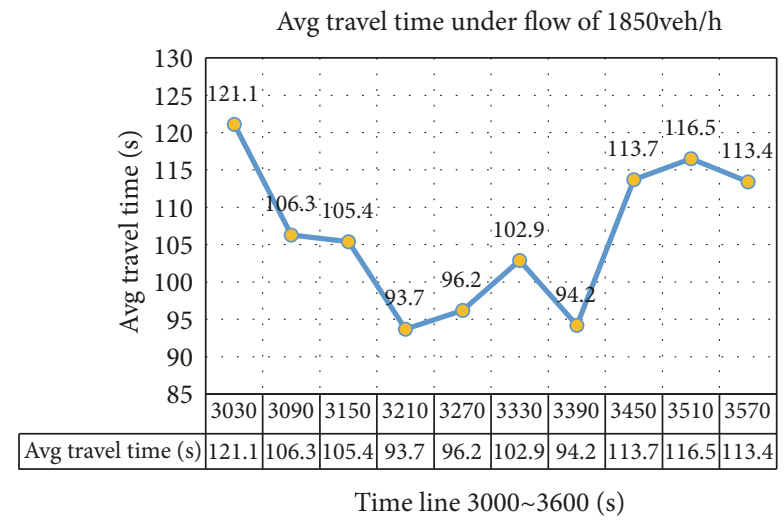

FIgURE 6: Travel time line chart (Flow 1850veh/h).

travel time changes in 3000 3600s. From Figures 3 and 4, we can see that the average driving time does not increase greatly from $400 v \mathrm{ve} / \mathrm{h}$ to $1200 \mathrm{veh} / \mathrm{h}$. When the flow increased from $1200 \mathrm{veh} / \mathrm{h}$ to $1550 \mathrm{veh} / \mathrm{h}$, the average driving time greatly increases, and traffic congestion occurs. From Figures 5 and 6 , when the flow increased from $1200 \mathrm{veh} / \mathrm{h}$ to $1550 \mathrm{veh} / \mathrm{h}$, the average travel time is increasing and the traffic congestion is more serious. In addition, with the increase of the traffic volume, the road impedance shows a certain random fluctuation along with the continuous rise of the traffic volume, and the fluctuation of the road impedance is more obvious with the increase of the traffic volume.

To avoid the existence of abnormal value leading to inaccuracy of result, we consider the distribution skewness in a group of data and its tail length to make the result more convincing. The processing and analysis of a group of simulation data extract the data of key positions such as the first quartile and the third quartile, D-value and deviation between the quartiles, and the mean of this group of data to compare the data to define the reliability of simulation data. The groups of simulation data and their key position data are as shown in Table 3.

Under $L_{a}=0.5 \mathrm{~km}, v_{f}=50 \mathrm{~km} / \mathrm{h}$, it is observed from the simulation data in Table 3 that the travel time of vehicle flow is increasing with the increase in the transportation volume of the stretch. In the meanwhile, the mean of travel time is $39.03 s>36 s$ when the transportation volume is $400 v e h / h$. In addition, $\mid \Delta_{a} /$ ave $\mid$ and $\mid \Delta_{b} /$ ave $\mid$ are basically smaller than 0.20 ; that is, the average travel time of vehicle flow for different transportation flows is all concentrated around the mean, indicating the deviation of data is small and the simulation data is effective.

The calculation result of the dynamic impedance function model equation is compared with the simulation result of this model, with result as shown in Figures 7 and 8.

It is observed from Figures 7 and 8 that the error rate between the simulation result and the calculation result of model is below $10 \%$ when the loaded transportation volume is below $1500 \mathrm{veh} / \mathrm{h}$. The error rate between the two jumps to $27.18 \%$ from $7.71 \%$ when the loaded transportation volume is increased from $1500 v e h / h$ to $1550 v e h / h$. Besides, the error rate is basically above $30 \%$ when the transportation volume is greater than $1600 \mathrm{veh} / \mathrm{h}$. The main reason responsible for this is that traffic jam occurs on the simulation stretch, transportation volume increases from $1500 \mathrm{veh} / \mathrm{h}$ to $1550 \mathrm{veh} / \mathrm{h}$, and the queuing vehicles have spread to the upstream junction, leading to sharp increase of target parameter. This indicates that this model has some limitation in defining the impedance of travel time of vehicle flow for the jammed stretch (complete jamming on the stretch). Spreading to the upstream junction, the error rate between the model and the impedance of travel time of jamming vehicle flow on the stretch whose jamming has not spread to the upstream junction is lower than $10 \%$. Thus, the dynamic impedance function model proposed can be well applied to define impedance of travel time of vehicle flow in the jamming condition, to provide a basis of path impedance for the dynamic distribution of traffic.

\section{Conclusion}

In order to solve the shortcomings of BPR function for the description of traffic flow microfeatures and to provide a basis for traffic distribution in future research, this paper proposed an Urban Road Dynamic Impedance Modeling. Firstly, dividing the road impedance into motion road impedance and the queue road impedance, the variation law of traffic 
TABLE 3: Simulation data unit(s).

\begin{tabular}{|c|c|c|c|c|c|c|c|}
\hline $\begin{array}{l}\text { Flow } \\
(\text { veh } / h) \\
\end{array}$ & $\begin{array}{l}\text { Avg. } \\
\text { (avg) }\end{array}$ & $\begin{array}{c}\text { Lower Quartile } \\
\left(Q_{1}\right) \\
\end{array}$ & $\begin{array}{c}\text { Upper Quartile } \\
\left(Q_{3}\right) \\
\end{array}$ & $\begin{array}{c}Q_{1}-\text { ave } \\
\left(\Delta_{\mathrm{Q} 1}\right)\end{array}$ & $\begin{array}{c}Q_{3}-a v e \\
\left(\Delta_{\mathrm{Q} 3}\right)\end{array}$ & $\begin{array}{c}\mid \Delta_{a} / \text { ave } \mid \\
(\%)\end{array}$ & $\begin{array}{c}\mid \Delta_{b} / \text { ave } \mid \\
(\%)\end{array}$ \\
\hline 400 & 39.03 & 35.95 & 42.60 & -3.08 & 3.57 & 7.9 & 9.1 \\
\hline 450 & 39.52 & 31.50 & 43.85 & -8.03 & 4.32 & 20.3 & 10.9 \\
\hline 500 & 40.07 & 36.35 & 43.70 & -3.72 & 3.63 & 9.3 & 9.1 \\
\hline 550 & 41.09 & 39.35 & 43.15 & -1.74 & 2.06 & 2 & 5.0 \\
\hline 600 & 41.98 & 37.75 & 46.15 & -23 & 17 & 10.1 & 9.9 \\
\hline 650 & 43.02 & 39.50 & 56.70 & -3.52 & 13.68 & 8.2 & 31.8 \\
\hline 700 & 43.93 & 36.80 & 54.35 & -7.13 & 10.42 & 16.2 & 23.7 \\
\hline 750 & 45.22 & 42.05 & 59.90 & -3.17 & 14.68 & 7.0 & 32.5 \\
\hline 800 & 46.32 & 42.50 & 50.80 & -3.82 & 4.48 & 8.2 & 9.7 \\
\hline 850 & 47.88 & 42.20 & 55.30 & -5.68 & 7.42 & 11.9 & 15.5 \\
\hline 900 & 48.91 & 43.75 & 53.80 & -5.16 & 4.98 & 11.8 & 9.1 \\
\hline 950 & 50.19 & 42.85 & 58.05 & -7.34 & 7.86 & 17.1 & 13.5 \\
\hline 1000 & 50.86 & 42.85 & 58.05 & -8.01 & 7.19 & 18.7 & 12.4 \\
\hline 1050 & 51.42 & 43.20 & 58.70 & -8.22 & 7.28 & 19.0 & 12.4 \\
\hline 1100 & 52.77 & 49.40 & 58.20 & -3.37 & 5.43 & 6.8 & 9.3 \\
\hline 1150 & 52.64 & 43.30 & 58.20 & -9.34 & 5.56 & 17.7 & 10.6 \\
\hline 1200 & 52.03 & 47.40 & 56.90 & -4.63 & 4.87 & 8.9 & 9.4 \\
\hline 1250 & 57.00 & 52.55 & 61.50 & -4.45 & 4.50 & 7.8 & 7.9 \\
\hline 1300 & 58.75 & 53.20 & 66.45 & -4.35 & 3.35 & 6.9 & 5.3 \\
\hline 1350 & 65.76 & 62.85 & 69.80 & -2.91 & 4.04 & 4.4 & 6.1 \\
\hline 1400 & 65.99 & 56.70 & 69.05 & -5.07 & 0.72 & 14.4 & 4.6 \\
\hline 1450 & 66.74 & 62.75 & 70.35 & -3.99 & 3.61 & 6.4 & 5.1 \\
\hline 1500 & 70.24 & 65.65 & 710 & -4.59 & 3.86 & 7.0 & 5.2 \\
\hline 1550 & 84.32 & 74.35 & 94.00 & -9.97 & 9.68 & 13.4 & 10.3 \\
\hline 1600 & 93.48 & 84.50 & 105.45 & -8.98 & 11.97 & 10.6 & 11.4 \\
\hline 1650 & 93.28 & 85.95 & 102.60 & -7.33 & 9.32 & 8.5 & 9.1 \\
\hline 1700 & 99.01 & 87.55 & 111.50 & -11.46 & 12.49 & 13.1 & 11.2 \\
\hline 1750 & 100.93 & 93.25 & 107.05 & -7.68 & 6.12 & 8.2 & 5.7 \\
\hline 1800 & 101.38 & 97.15 & 106.75 & -23 & 5.37 & 4.4 & 5.0 \\
\hline 1850 & 106.34 & 95.20 & 115.10 & -11.14 & 8.76 & 10.5 & 8.2 \\
\hline$\ldots$ & $\ldots$ & $\ldots$ & $\ldots$ & $\ldots$ & $\ldots$ & $\ldots$ & $\ldots$ \\
\hline
\end{tabular}

impedance at the microlevel is found. Then, by using the relationship between traffic flow, queue length and road travel time, the urban road dynamic impedance modeling was constructed. Finally, the effectiveness of the model in the dynamic changing environment of traffic flow is verified by simulation analysis.

Simulation results show that the estimation error of impedance (travel time) of the proposed algorithm is less than $10 \%$, when the road is not completely blocked. Therefore, dynamic traffic impedance model can adapt to real-time road impedance estimation in free states and some road congestion states. It also makes up for the deficiency of the existing studies on the change laws of microscopic traffic impedance and improves the comprehensiveness, timeliness, and portability of the model.

However, there is still a large error in the estimation of completely blocked sections, so it is necessary to further study the estimation of road impedance under saturated or oversaturated traffic density. The simulation results may also be affected by environmental factors, such as weather, temperature, road conditions, and emergencies, which have a certain impact on the results. This will be the direction of our research for the next period of time.

\section{Data Availability}

The data used to support the findings of this study are included within the article.

\section{Conflicts of Interest}

The authors declare that there are no conflicts of interest regarding the publication of this paper. 


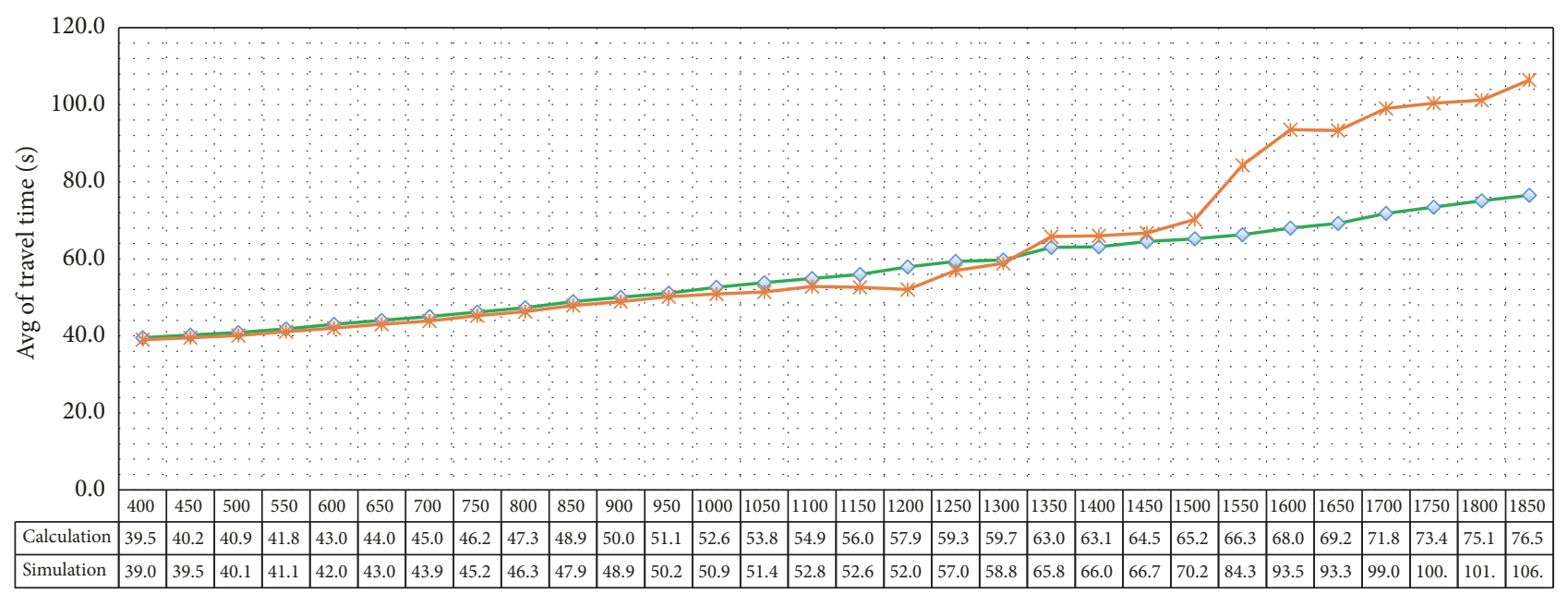

Traffic flow loaded (veh/h)

$\checkmark$ Calculation

* Simulation

Figure 7: Comparison between model calculation results and simulation results.

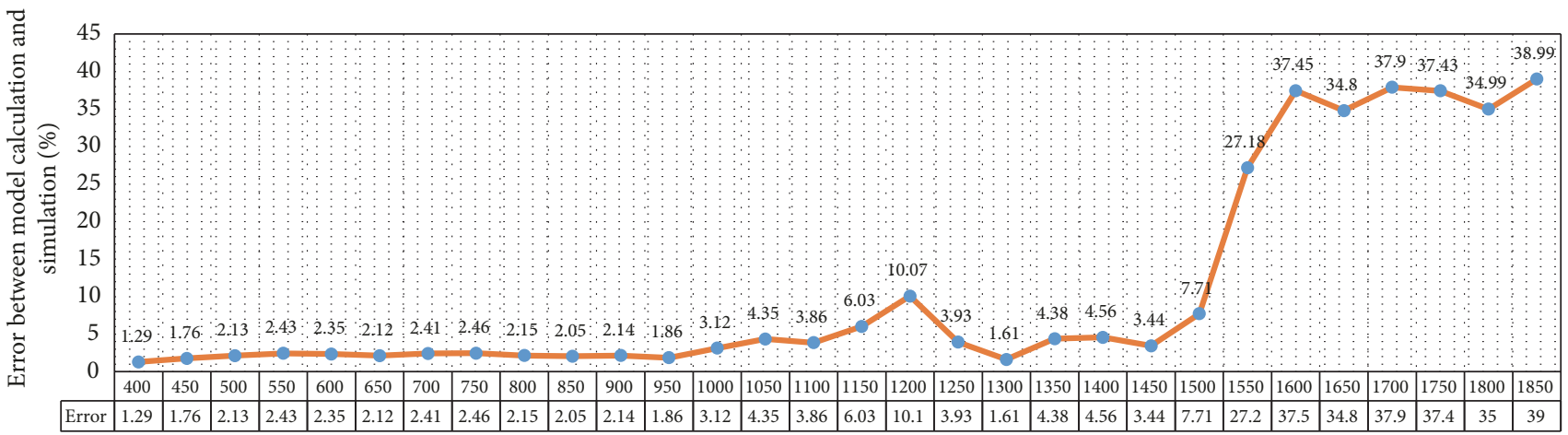

Traffic flow loaded (veh/h)

FIGURE 8: Error distribution between model calculation results and simulation results.

\section{Acknowledgments}

This work was partially supported by the Jiangxi Provincial Department of Education Science Research Fund Project (Grant no. GJJ170420), Jiangxi Provincial Department of Transport Science Research Fund Project (Grant no. 2018X0015), and ECJTU “TIANYOU Talent” Development Program (Grant no. 201709). The authors would like to thank Dr. Liqun Peng from East China Jiao Tong University for his valuable opinions and suggestions for this paper.

\section{References}

[1] X. T. Wang, Y. L. Zhang, and P. Zhang, "Traffic signal optimization based on system equilibrium and bi-level multi-objective programming model," in Proceedings of the International Conference on Green Intelligent Transportation System and Safety, vol. 419, pp. 429-438, 2017.

[2] M. Venigalla, X. Zhou, and S. Zhu, "Psychology of route choice in familiar networks: Minimizing turns and embracing signals," Journal of Urban Planning and Development, vol. 143, no. 2, 2016.
[3] Z. W. Qu, Y. Xing, and X. M. Song, "A study on the coordination of urban traffic control and traffic assignment," Discrete Dynamics in Nature Society, vol. 2012, Article ID 367468, 12 pages, 2012.

[4] L. Yan, B. Liu, and J. Lu, "Comprehensive model of traffic impedance on urban arterial road with signalized intersection," in Proceedings of the CICTP 2012: Multimodal Transportation Systems-Convenient, Safe, Cost-Effective, Efficient, pp. 503509, 2012.

[5] J. Dong and G. J. Shen, "A weight-based road impedance function model," Advanced Materials Research, vol. 756-759, pp. 2750-2755, 2013.

[6] Manual, Highway Capacity. "HCM2010.” Transportation Research Board, National Research Council, Washington, DC, USA, 2010.

[7] H. Spiess, "Conical volume-delay functions," Transportation Science, vol. 24, no. 2, pp. 153-158, 1990.

[8] I. M. Zudhy, T. Sumi, and A. Munawar, "Implementation of the 1997 Indonesian Highway Capacity Manual (MKJI) volume delay function," Journal of the Eastern Asia Society for Transportation Studies, vol. 7, pp. 350-360, 2010. 
[9] H. Tan, Y. Yang, and L. Zhang, "Improved BPR function to counter road impedance through OD matrix estimation of freight transportation," Journal of Highway and Transportation Research and Development (English Edition), vol. 11, no. 2, pp. 97-102, 2017.

[10] L. Ning, S. C. Zhao, and H. Nan, "Further study of impedance function based on BPR function," Journal of Wuhan University of Technology: Transportation Science \& Engineering, vol. 37, pp. 545-548, 2013.

[11] A. Sadeghi-Niaraki, M. Varshosaz, K. Kim, and J. J. Jung, "Real world representation of a road network for route planning in GIS," Expert Systems with Applications, vol. 38, no. 10, pp. 1199912008, 2011.

[12] Y. Luo, N. Wang, H. Xiang et al., "Road impedance model study under the control of intersection signal," Mathematical Problems in Engineering, vol. 2015, Article ID 468943, 6 pages, 2015.

[13] N. He and S. Zhao, "Discussion on Influencing Factors of Freeflow Travel Time in Road Traffic Impedance Function," Procedia - Social and Behavioral Sciences, vol. 96, pp. 90-97, 2013.

[14] Q. Cai, Y. Chen, H. Li, and J. Du, "Traffic Impedance Based on Grey Mean Generating Function," Advanced Science Letters, vol. 19, no. 4, pp. 1136-1140, 2013.

[15] M. X. Gao and J. W. Mo, "A quickest flow model for evacuation route and departure schedule planning," in Proceedings of the 2012 Second International Conference on Electric Technology and Civil Engineering, pp. 158-161, IEEE Computer Society, 2012.

[16] W. Jin, Q. Gan, and J. Lebacque, "A kinematic wave theory of capacity drop," Transportation Research Part B: Methodological, vol. 81, pp. 316-329, 2015.

[17] J. Sun, B. Pan, K. Li, and H. Zhang, "Method for dynamic measuring of delay at unsaturated signalized intersections," Journal of Tongji University, vol. 39, no. 7, pp. 1007-1012, 2011.

[18] Z. Qu, X. Yang, and X. Song, "A study on the coordination of urban traffic control and traffic assignment," Discrete Dynamics in Nature and Society, vol. 2012, Article ID 367468, 12 pages, 2012. 


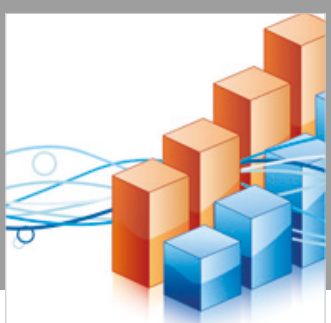

Advances in

Operations Research

\section{-n-m}
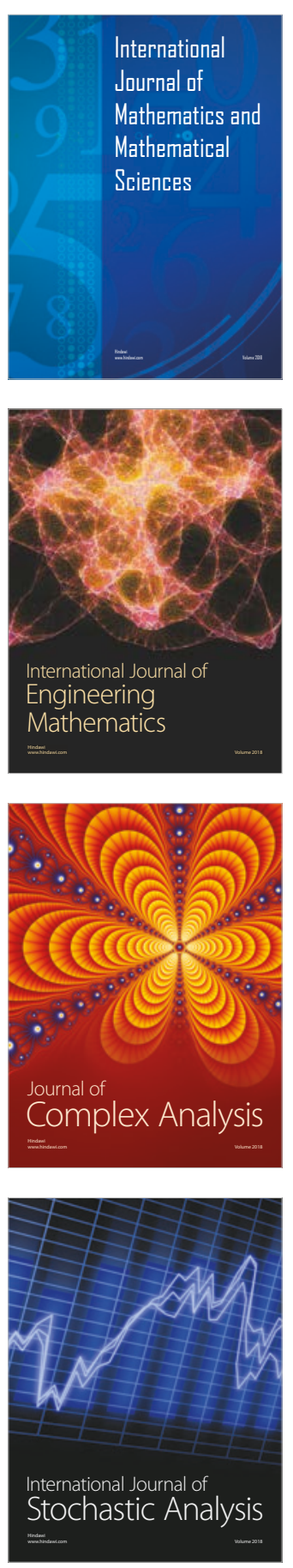
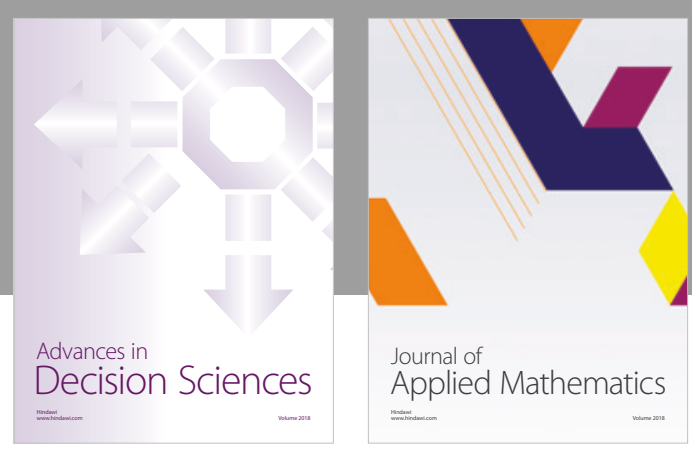

Journal of

Applied Mathematics
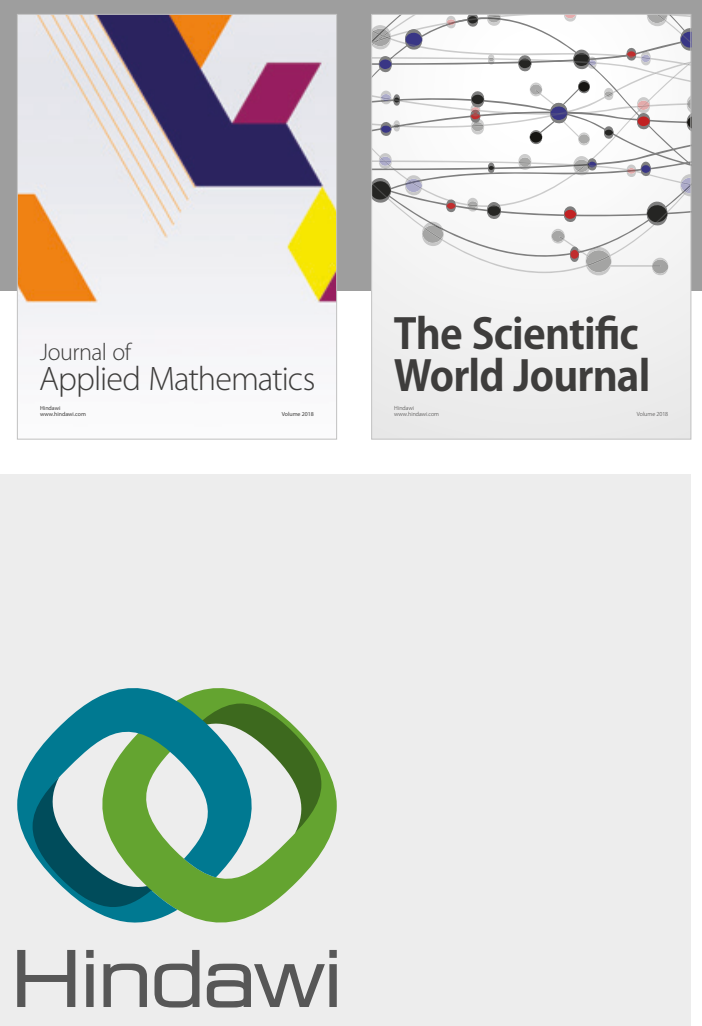

Submit your manuscripts at

www.hindawi.com

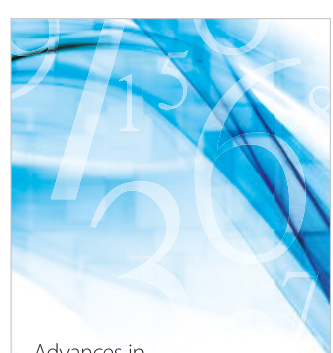

Advances in
Numerical Analysis
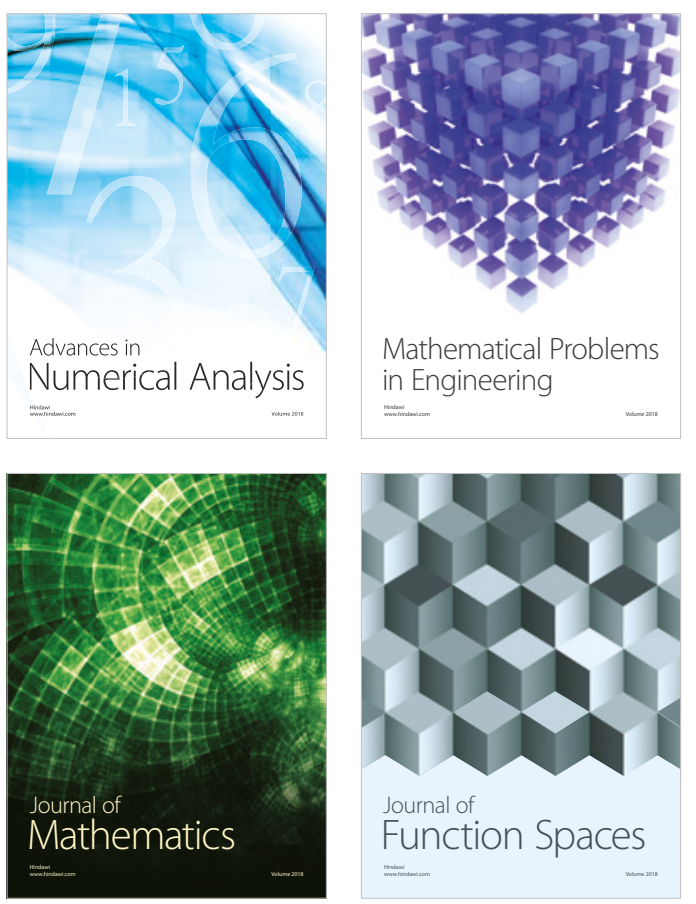

Mathematical Problems in Engineering

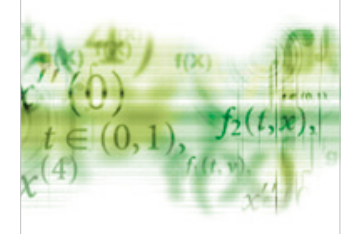

International Journal of

Differential Equations

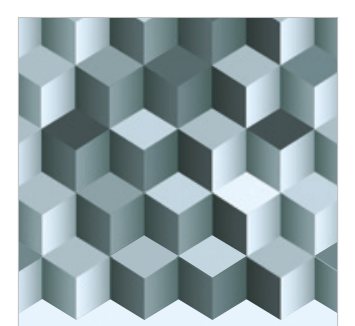

Journal of

Function Spaces

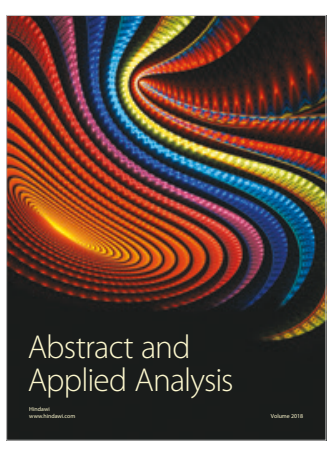

The Scientific

World Journal

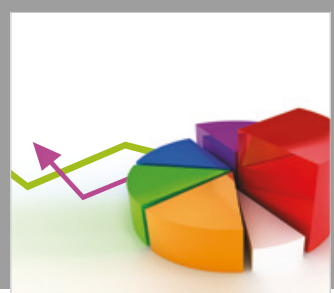

Journal of

Probability and Statistics
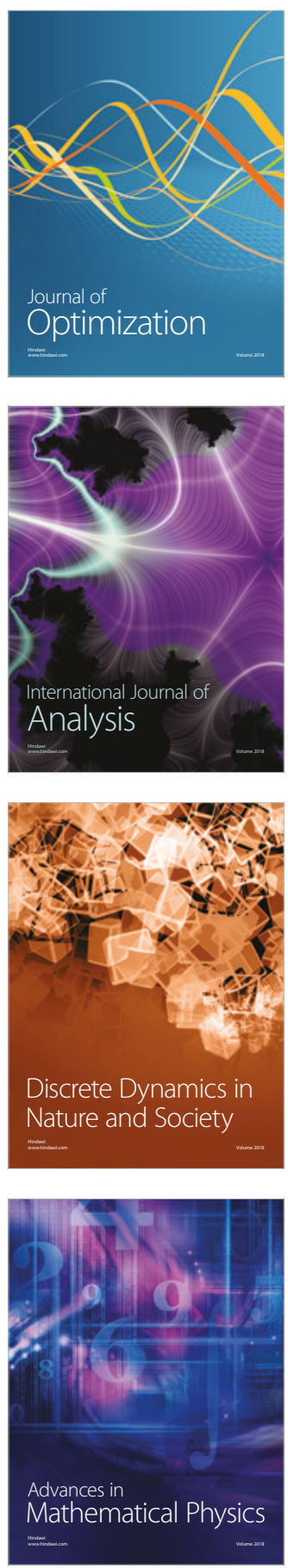